\title{
Effect of possible rotor deformation on the probability of face contact for a liquid film bearing
}

\author{
N. Y. Bailey ${ }^{\mathrm{a}, \mathrm{b}}$, S. Hibberd ${ }^{\mathrm{b}}$, H. Power ${ }^{\mathrm{c}, 1}$ \\ ${ }^{a}$ Department of Mechanical Engineering, University of Bath, Bath, BA2 7AY. UK. \\ ${ }^{b}$ Mathematical Sciences, University of Nottingham, Nottingham, NG7 2RD. UK. \\ ${ }^{c}$ Faculty of Engineering, University of Nottingham, Nottingham, NG7 2RD. UK.
}

\begin{abstract}
The possibility of face contact is examined for a coaxial rotor-stator bearing in dynamic motion constrained by a highly rotating very thin liquid film. A modified Reynolds equation for pressurised flow is coupled to the bearing structure leading to determination of the bearing gap from solving a nonlinear secondorder non-autonomous ordinary differential equation. Periodic solutions are found via a mapping solver. Rotor deformation is parametrised by a coning angle and considered a random variable. The method of derived distributions is used to quantify variation in coning angle and examine the probability of rotorstator contact. Additionally, effects of possible destabilising random aspects on the axial rotor oscillations are investigated. Exact solutions for probability of contact are obtained for various bearing configurations.
\end{abstract}

Keywords: Reynolds equation, method of derived distribution, probability density function, face contact

\section{Introduction}

Liquid film bearings typically comprise a coaxial axisymmetric rotor and stator pair with a thin liquid film used to maintain a clearance between the faces when the bearing experiences external loading. The local film pressure may be enhanced through normal motion of the plates and flow dynamics associated with the bearings rotational motion. Commonly industrial applications of this type of bearing are required to operate with increasing rotor speed; correspondingly inertia effects which are typically neglected in classical lubrication

Email address: henry.power@nottingham.ac.uk (H. Power ) 
theory need to be considered. In this high speed scenario, comprehensive and accurate predictions of variation in the face clearance is essential to examine the feasibility of contact between the bearing faces.

For current advanced applications the use of a coned face can be incorporated in the bearing design to further increase the lubrication force, enabling a design film thickness to be maintained. Multiple lubrication theory studies have identified an optimal coning angle and bearing conditions to maximise the stability [1], [2] and critical angle to avoid face contact [3].

Alternatively, dynamic coned rotor configuration may arise by over- pressurisation of the flow through the bearing structure, inducing structural displacement. In a previous study [4] such bearing dynamics were examined for prescribed periodic axial rotor oscillations where a no-slip boundary condition was imposed on the bearing faces. Results indicate the film thickness can become increasingly small reaching magnitude of the order of the surface roughness.

To evaluate the case of very small liquid films, comparable to the order of magnitude of the irregular surface roughness, exisiting numerical simulation becomes impractical on account of applying a no-slip boundary condition on the irregular surface. To extend the modelling approach an effective slip velocity condition can be implemented at a suitable equivalent smooth surface. Such an approach is characterised by a slip length parameter having the same order of magnitude as the surface roughness [5], [6]. A bearing evaluation for a slip condition is incorporated in the dynamic analysis of a high speed thrust bearing, for parallel faces [7] and a coned rotor [8] under prescribed axial oscillations of the rotor. These papers model the bearing dynamics under external loading where the fluid gap can attain thickness of nano or micro scale size. Two different flow conditions are considered; one corresponds to the flow being driven purely from the rotation of the rotor when the stator is stationary, usually referred to as the stationary or steady state condition in the literature. The other case considered is that of the rotating face (rotor) undergoing prescribed periodic axial oscillations where the stator also moves axially in response to the resulting film dynamics, referred to as a dynamic bearing. For both flow conditions, the limiting case of a very small fluid film is considered, where a slip boundary condition is included in order to account for the interaction of the flow field with the surface roughness. Centrifugal inertia effects are also taken into account which are not included in the classical lubrication theory, but can be important for high rotational operational speeds. In the case of a steady state condition analytical solutions of the problem are reported in [7] and [8], which are consistent with the classical no-slip solution of Kalita et al. [9], when the slip condition is not considered (see [10] for the corresponding experimental verification). Even in this simple flow configuration, the bearing behaviour is defined by the complex interaction between the different parameters defining the problem; bearing geometry, rotor inclination angle, fluid pressurisation, slip condition and effects of inertia. In particular for an externally pressurised bearing, the effects of inertia have an opposite effect to those of the imposed pressure gradient, where a critical value of a centrifugal inertia parameter can be found, dependant on the bearing geometry, where a zero flux condition through the bearing is achieved 
(for more details see [8]). The predicted steady state bearing behaviour is also consistent with the experimental observation reported in Lee et al. [11], where two additional issues were identified; combinations of the seal parameters that maximise the fluid film stiffness is optimal for safe non-contacting operation, and for high rotational speed applications it is necessary to account for the vibration of other seal and system components. The first observation is consistent with the analytical prediction of the steady state conditions and is also observed during the dynamic behaviour where the maximum values of the fluid film are found at larger values of the film stiffness, see Figure 4 in [8]. The second observation is the main reason why the dynamic bearing behaviour is being studied, which is essential in the present work.

Due to the complexity of the fluid-structure interaction, the case of a dynamic bearing requires the resulting coupled system of equations to be solved numerically. The numerical simulation of the flow field incorporates a slip velocity boundary condition in order to characterize the surface roughness of the solid boundaries (rotor and stator) predicts a significant effect of this condition on the resulting flow. In these cases the bearing faces are predicted to not have contact in the case of highly rotating parallel bearings, although the fluid film can become very small during its dynamic behaviour. Notably for the case of a coned bearing geometry incorporating a slip boundary condition, face contact is possible with significant practical implications. A further restriction was that all parameters and variables in these articles were deterministic defined, which in many cases are not accurately known. In this current work, further analysis evaluates the uncertainty associated with the values of key bearing quantities.

Improved predictability of bearing dynamics is of increased interest, especially the quantification of uncertain effects. A parallel face bearing may have rotor deformation due to over-pressurisation, which are described in this paper by a conical shape with coning angle obtained according to the imposed pressure field. The magnitude of the corresponding conical angle is difficult to determine and considered as a random variable. Predictions from an earlier study [8] highlights undesirable aspects under extreme conditions for a liquid gap of nano or micro scale including possible stator-rotor collision. An additional complementary source of uncertainty in a bearing model is the random axial motion of the rotor due to external excitations. This study quantifies the effects of variability in the coning angle and amplitude of rotor oscillations on the probability of face contact.

A general approach to dynamical systems with uncertainty that arises from external sources, leading to the excitation of the system, are typically based on the Monte Carlo method. In the Monte-Carlo method, samples from the input parameter are considered to be uncertain and for each different value of input, the deterministic model is run to give the corresponding output. It is a robust method and can be used on complex problems, including with a high number of uncertain parameters. However for results to have a sufficient level of accuracy, typically an extensive number of runs (Monte Carlo realisations) are needed. To increase efficiency for quantifying uncertainty, a number methods have been developed, for example the Markov Chain Monte Carlo method, Gaussian process 
emulator and polynomial chaos, among others.

A simplified approach to model the external excitations was formulated by Hou et al. [12] where the excitations where assumed to be periodic with a random amplitude. This was incorporated into a stochastic model for a linear single degree of freedom system to examine the stationary moment responses. An approach to quantify effects of uncertain parameters on the response of a dynamic rotor bearing system was investigated by Zigang et al. [13]. Governing equations were derived to include uncertainty in the nonlinear support stiffness and damping through stochastic modelling; results achieved by an adapted polynomial chaos expansion technique are shown to agree well with those obtained via a Monte-Carlo simulation. The dynamic behaviour and response statistics of the stochastic system were investigated through the probability density function and mean value. Results confirmed that predictions are notably effected by the uncertainty in the damping and nonlinear support stiffness values.

The method of derived distributions [14] is an alternative approach that can be used to exactly quantify the effects of uncertainty in the input parameters when a deterministic mathematical model of the problem in consideration exists. On describing the random input parameter by a probability density function, defining the relation between the deterministic values of the input and corresponding output parameters, the probability density function and the cumulative distribution function of the output can be exactly computed. This methodology was implemented by Bailey et al. [15] to quantify the impact of variability in the slip condition and the axial displacement of the rotor due to external excitations. These parameters where considered as random variables, due to uncertainty in their values, and the probability of contact is analysed from a corresponding exact solution.

The methodology in Bailey et al. [15] is used in this paper to investigate the probability of contact due to uncertainty in the magnitude of the deformed conical angle and amplitude of prescribed periodic axial oscillations of the rotor. Prior to contact, the bearing thicknesses may be of nano and micro scales, and correspondingly a slip length of the order of the surface roughness are considered. These important extensions give a criterion for controlling or mitigate the likelihood of possible bearing contact. In Section 2 a deterministic mathematical model is derived for a fluid film coned bearing incorporating a slip boundary condition and prescribed periodic axial oscillations of the rotor. The high-speed rotational bearing operation is incorporated through centrifugal fluid inertia as used in Bailey et al. [8]. A modified Reynolds equation is formulated and coupled to generic structural dynamics for a bearing.

The resulting pressure field, solution of the Reynolds equation, exerts an axial force on the stator that can induce its displacement as a reaction to the enforced rotor motion, the corresponding bearing dynamics are derived resulting in a single nonlinear second-order non-autonomous ordinary differential equation for the face clearance. Periodic bearing solutions are obtained iteratively via a mapping solver, similar to the stochastic algorithm developed in [15]. Results are presented in Section 3, initially with deterministic results of the bearing behaviour, and used for later calculations for the probability of face contact. 


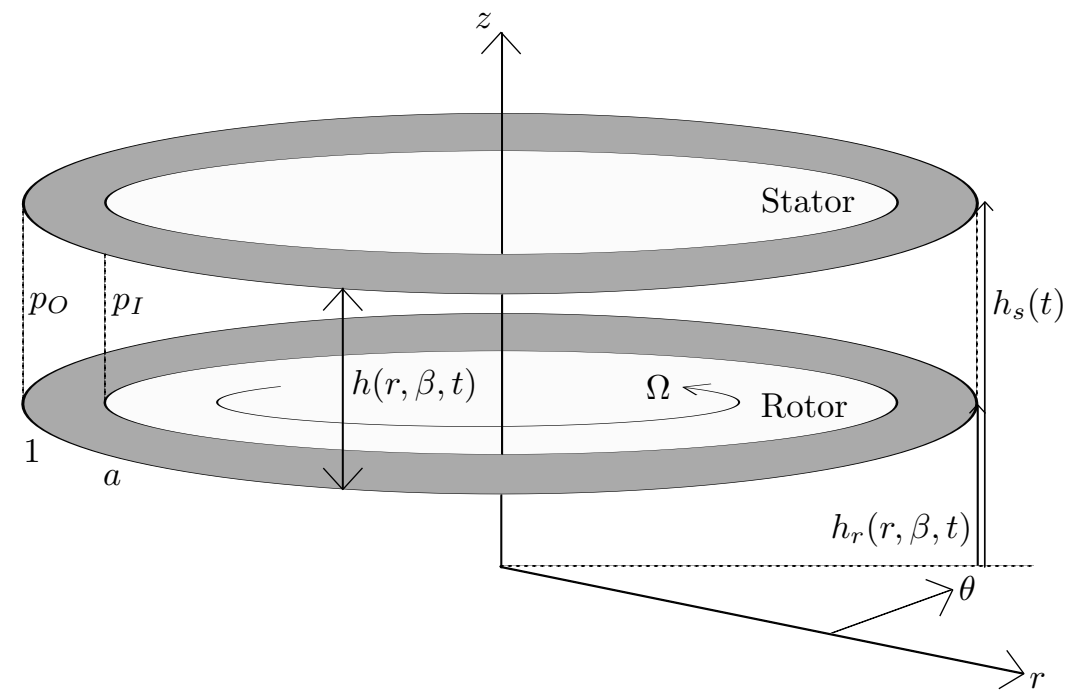

Figure 1: Geometry of a fluid-lubricated thrust bearing in an axisymmetric dimensional cylindrical polar coordinate system $(r, \theta, z)$.

In Section 3.2 the probability of contact is investigated incorporating the lack of precise knowledge in the coning angle. In this analysis the coning angle is taken as a random variable with prescribed mean and standard deviation. The effect on the probability of contact due to variability in both the coning angle and amplitude of rotor oscillations is examined in Section 3.3 through taking the corresponding parameters as random variables with prescribed mean and standard deviation. Finally a parametric study examines the effect on the probability of contact of core design geometries and operational conditions.

\section{Mathematical Model}

A high speed liquid film bearing model, including a slip boundary condition, is taken from the simplified model in Bailey et al. [8]. The incompressible Navier-Stokes momentum and continuity equations are used to develop a fluid flow model in axisymmetrical coordinates as illustrated in Figure 1, incorporating a coned rotor in close proximity to a plain stator. The mathematical formulation is expressed in dimensionless variables using typical values of a characteristic bearing film thickness $\hat{h}_{0}$, radius $\hat{r}_{0}$ and rotor velocity $\hat{\Omega} \hat{r}$ to give the dimensionless slip length as $l_{s}=\hat{l}_{s} / \hat{h}_{0}$ and the aspect ratio $\delta_{0}=\hat{h}_{0} / \hat{r}_{0}$ as being small $\delta_{0} \ll 1$ for a thin film bearing. The dimensionless radius $r=\hat{r} / \hat{r}_{0}$ and height $z=\hat{z} / \hat{h}_{0}$ are identified and the dimensionless time variable is defined by $t=\hat{\omega} \hat{t}$, with $\hat{\omega}$ denoting the angular frequency. The dimensionless velocities are given by $\hat{u} / \hat{U}, \hat{v} / \hat{\Omega} \hat{r}_{0}$ and $\hat{w} / \hat{h}_{0} \hat{T}^{-1}$. 

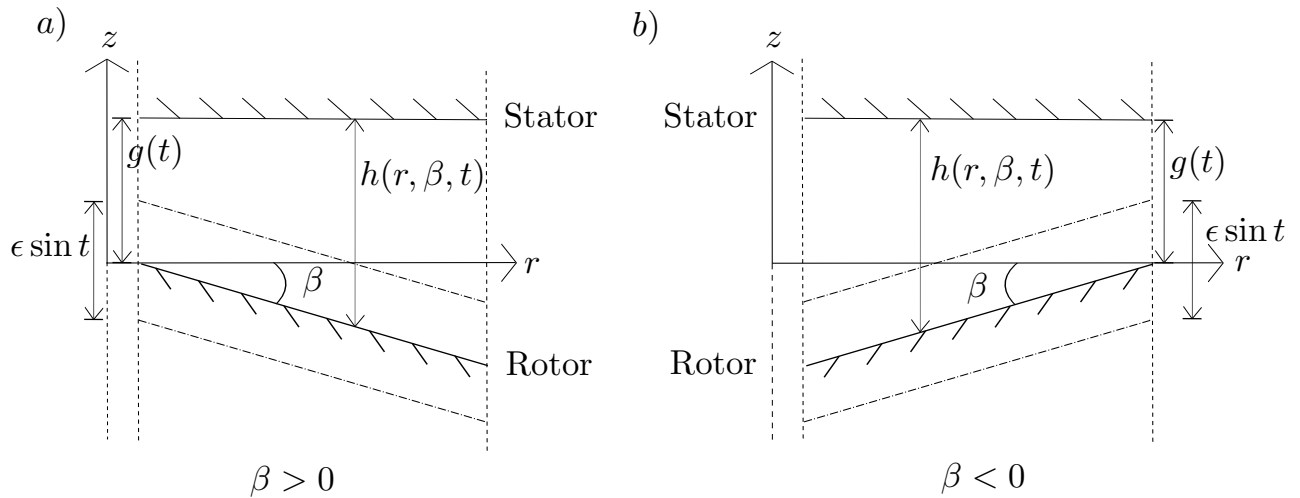

Figure 2: Schematic of an axisymmetric bearing geometry in the $z-r$ plane in the case of a) PCB $\beta>0$ and b) NCB $\beta<0 ; h$ denotes the film thickness and $g$ the minimum face clearance.

Pressures are imposed at the inner radius $\hat{r}_{I}$ and outer radius $\hat{r}_{O}$, which drives a radial flow and gives the characteristic pressure as $\hat{P}$, resulting in inner and outer dimensionless pressurisations $p_{I}=\hat{p_{I}} / \hat{P}$ and $p_{O}=\hat{p_{O}} / \hat{P}$, respectively with dimensionless atmospheric pressure $p_{a}=\hat{p_{a}} / \hat{P}$. Over-pressurisation of the bearing can lead to a fixed deformation of the rotor, giving a coning angle $\hat{\beta}$, as shown in Figure 2. The value of coning is taken as not known exactly, but sufficiently small for $\cos \hat{\beta} \simeq 1$ and $\sin \hat{\beta} \simeq \hat{\beta}$. Internal pressurisation $\left(p_{I}>p_{O}\right)$ results in a positively coned bearing $(\mathrm{PCB})$ and external pressurisation $\left(p_{O}>\right.$ $\left.p_{I}\right)$ in a negatively coned bearing (NCB) as illustrated in Figure 2. The cases of a $\mathrm{PCB}$ and $\mathrm{NCB}$ are considered separately with a coning angle scaled by $\beta=\hat{\beta} / \delta_{0}$ with $\beta=O(1)$ at most ensuring a lubrication approximation remains valid.

The dimensionless film thickness for a coned bearing subject to prescribed periodic axial oscillations $h_{r}(t)$ of magnitude $\epsilon \sin t$ is given by

$$
\begin{array}{lll}
h(r, \beta, t)=h_{s}(t)-h_{r}(r, \beta, t)=h_{s}(t)-\epsilon \sin t+(r-a) \beta & \text { if } \quad & \beta \geq 0, \\
h(r, \beta, t)=h_{s}(t)-h_{r}(r, \beta, t)=h_{s}(t)-\epsilon \sin t+(r-1) \beta & \text { if } & \beta<0 .
\end{array}
$$

In (1) the axial height of the stator is denoted by $h_{s}(t)$ and $\epsilon$ as a measure of the axial displacement amplitude. For convenience the outer radius is scaled to 1 and the inner radius to $a=\hat{r}_{I} / \hat{r}_{0}$, with $a \leq r \leq 1$. The rotor height $h_{r}$ has the temporal and spatial dependence independent of each other, giving the film thickness $h$ as the stator height minus the axial displacement from the prescribed oscillations of the rotor plus or minus the axial height change across the bearing due to the coning angle for a $\mathrm{PCB}$ or $\mathrm{NCB}$, respectively.

The radial Reynolds number is defined as $R e_{U}=\hat{\rho} \hat{h}_{0} \hat{U} / \hat{\mu}$, with dynamic viscosity $\hat{\mu}$ and density $\hat{\rho}$; classical lubrication theory neglects inertia as $\operatorname{Re}_{U} \delta_{0} \ll 1$. To include very high speed bearing operation the current work retains centrifugal 
inertia, in this case expressions of the order $R e_{U} \delta_{0}\left(R e^{*}\right)^{2}$ are assumed of $O(1)$, with $\left(R e^{*}\right)^{2}=\left(\hat{\Omega} \hat{r}_{0} / \hat{U}\right)^{2} \gg 1$. To retain the effects of the pressure gradient at leading order the pressure is scaled by $\hat{P}=\hat{\mu} \hat{r}_{0} \hat{U} / \hat{h}_{0}^{2}$ and time dependence effects are parametrised by the squeeze number $\tilde{\sigma}=\hat{r}_{0} /(\hat{U} \hat{T})$. The Froude number $F r=\hat{U}\left(\tilde{g} \hat{h}_{0}\right)^{-1 / 2}$ characterises the relative significance of gravitational effects relative to the radial inertia effects, where the acceleration due to gravity is denoted by $\tilde{g}$. However, if $\operatorname{Re}_{U} \delta_{0} F r^{-2} \ll 1$, with the Froude number is $O(1)$ to ensure consistency with lubrication theory, gravity may be neglected.

For a bearing with a narrow gap, the governing equation for the incompressible fluid flow incorporating slip boundary conditions (see [8], [15]) is obtained from the leading order thin film approximation of the Navier-Stokes continuity equation, where terms of $O\left(\delta_{0}\right)$ are neglected. This leads to a modified Reynolds equation for slip flow

$$
\begin{aligned}
& \sigma \frac{\partial h}{\partial t}-\frac{1}{r} \frac{\partial}{\partial r}\left(r h^{2} \frac{\partial p}{\partial r}\left(h+6 l_{s}\right)\right) \\
& +\frac{\lambda}{r} \frac{\partial}{\partial r}\left(\frac{r^{2} h^{2}}{\left(h+2 l_{s}\right)^{2}}\left(h^{3}+10 h^{2} l_{s}+\frac{70}{3} h l_{s}^{2}+20 l_{s}^{3}\right)\right)=0,
\end{aligned}
$$

with scaled squeeze number $\sigma=12 \tilde{\sigma}$ and speed parameter $\lambda=3 R e_{U} \delta_{0}\left(R e^{*}\right)^{2} / 10$ characterising the importance of centrifugal inertia. If the speed parameter is small $\lambda \ll 1$, the effects of inertia are negligible, however the effect of relative rotational motion between the rotor and stator is still included through the velocity boundary conditions.

Using the approach in Bailey et al. [8] the modified Reynolds equation (2) can be solved analytically for the pressure field. The pressure field is then coupled to the stator axial displacement which is modelled via a spring-massdamper model. Defining the time dependent minimum face clearance (MFC) by

$$
\begin{gathered}
g(t)=h_{s}(t)-h_{r}(a, t)=h(a, t)-(r-a) \beta \quad \text { if } \quad \beta>0, \\
g(t)=h_{s}(t)-h_{r}(1, t)=h(1, t)-(r-1) \beta \quad \text { if } \quad \beta<0,
\end{gathered}
$$

as shown in Figure 2, with $g=\tilde{g} / \hat{h}_{0}$, a single ordinary differential equation is derived for the MFC

$$
\frac{\mathrm{d}^{2} g}{\mathrm{~d} t^{2}}+D\left(g, l_{s}, \beta\right) \frac{\mathrm{d} g}{\mathrm{~d} t}+S\left(g, \lambda, l_{s}, \beta\right)=\epsilon\left(\left(1-K_{z}\right) \sin t-D_{a} \cos t\right),
$$

with coefficients

$$
\begin{aligned}
& D\left(g, l_{s}, \beta\right)=D_{a}-\alpha \pi B\left(g, l_{s}, \beta\right), \\
& S\left(g, \lambda, l_{s}, \beta\right)=K_{z}(g-1)-\alpha \pi A\left(g, \lambda, l_{s}, \beta\right),
\end{aligned}
$$

for a PCB. Details are provided in [8], [15] where the equivalent equations for NCB are also provided. In equation (5), $D_{a}=\hat{D}_{a} R e_{U} \delta_{0} \mu \hat{T} / \hat{m} \hat{\rho}_{0} \hat{h}_{0}^{2}$ denotes the dimensionless structural damping coefficient, $K_{z}=\hat{K}_{z} R e_{U}{ }^{2} \delta_{0}{ }^{2} \mu^{2} \hat{T}^{2} / \hat{m} \hat{\rho}_{0}^{2} \hat{h}_{0}^{4}$ 
represents the restoring force coefficient (stiffness parameter) and $\alpha=\hat{\mu} \hat{U} / \hat{m} \hat{\omega}^{2} \delta_{0}^{3}$ is the force coupling parameter. Expressions for $A$ and $B$ are given in Appendix A, equations (A.1) and (A.2), respectively. The associated case for a NCB has the same form as equation (4), but modified effective restoring force term given by $S\left(g, \lambda, l_{s}, \beta\right)=K_{z}(g-(1-a) \beta-1)-\alpha \pi A\left(g, \lambda, l_{s}, \beta\right)$. Parameters for the coning angle $\beta$ and amplitude of rotor oscillations $\epsilon$ are considered as random variables to be analysed.

To solve the governing equation for the minimum face clearance (5), a stroboscopic map is formulated and Newton's method is used to obtain periodic solutions, following the approach used by Bailey et al. [15]. In contrast to the evaluation described in [15] the current study examines the important effect of increasing values of the coning angle $\beta$. The Euler scheme (parameter continuation) is used to find successive new initial conditions for increasing magnitude of coning angle $\beta+\triangle \beta$, using derivatives taken with respect to the coning angle. To compute the threshold values of the coning angle corresponding to a prescribed value of $g_{m i n}$, the formulated Euler scheme is directly extended; the limiting case of contact given by $g_{\min }=0$. The methodology used is the same as in Bailey et al. [15], but with the coning angle $\beta$ taken as a new dependent variable in the Newton scheme, instead of the slip length. Therefore the additional derivatives in the Jacobian are with respect to the coning angle instead of the slip length.

Detailed studies (numerical or experimental) of the above dynamic problem in the limit of large destabilising rotor oscillations which can result in possible face contact (rotor-stator) are scarce in the literature. The condition of very small face clearance requires a high order continuous numerical algorithm, such as the stroboscopic map formulation and Newton's method described in [15] which is also used in the current work, in order to be able to predict such small fluid clearances. Garratt et al. [16] examined numerical solutions of a dynamic bearing, using a less robust numerical scheme than the one employed in this work, to investigate cases when the rotor undergoes prescribed periodic axial oscillations of amplitude smaller than the equilibrium film thickness. Results showed the stator response was such that a finite face clearance is always guaranteed, avoiding possible face contact. Most of the studies in the literature examining dynamic behaviour are concerned with the instability conditions or possible ways of controlling them, where small periodic perturbation are imposed on steady state conditions, enabling possible growth or decay of the imposed amplitudes (eigenvalue problem) and the corresponding stability of the system to be predicted (for more details see [17]). On the other hand, experimental difficulties are known to occur at very small face clearances, of the nano or micro scale. Pan et al. [18] used a sphere glued to a cantilever to measure the damping force versus the film gap between the sphere and substrate, the sphere was forced to oscillate periodically with a prescribed amplitude with the aim of demonstrating the slip length was independent of the amplitude of the cantilever oscillation, i.e. constant slip length. The motion of the cantilever was described by a spring-mass-damper equation with the driven force given by a lubrication approximation of the flow around the sphere with a slip boundary 
condition, resulting in an equation which is mathematically equivalent to the stator displacement equation describing the present bearing problem. However in the cantilever problem the substrate is fixed, while in the present case the stator is allowed to move in response to the fluid film dynamics and motion of the rotor. Although these two problems are not equivalent, the cantilever experiments experienced significant difficulties in regions of very small fluid gap, mainly because the probe becomes overdamped near probe plate contact with a very large lubrication force making the measurements unreliable. Similar experimental difficulties are encountered in the experimental measurements of the dynamic bearing at very small gaps. In Zou et al. [19], the dynamics of a noncontacting mechanical face seal with a flexibly mounted rotor was studied numerically and experimental. The dynamics were investigated by considered both rotor-stator angular deformation and rotor-stator misalignment due to the response from the introduced rotor oscillations. The flow clearance between the faces was measured by proximity probes and varied through a pneumatic adjustment mechanism. While it was not possible to directly observe contact conditions, they were determined phenomenologically from pattern recognition of the probe signals and their power spectrum densities, as well as angular misalignment orbit plots, which were all calculated and displayed in real-time.

\section{Results}

\subsection{Deterministic Results}

Over-pressurisation of the bearing, internal pressurisation $\left(p_{I}>p_{O}\right)$ will produce a PCB and external pressurisation $\left(p_{O}>p_{I}\right)$ a NCB; the two cases of pressurisation are considered separately. The pressure imposed at the outer radius is taken as a base pressure $\left(p_{O}=p_{a}=1\right)$ and the pressure imposed at the inner radius is $p_{I}=1.5$ for internal pressurisation and $p_{I}=0.5$ for external pressurisation. The structural parameters used correspond to a dimensional structural stiffness $\hat{K}_{z}=5 \times 10^{6} \mathrm{Nm}^{-1}$ and damping $\hat{D}_{a}=300 \mathrm{Nsm}^{-1}$. Initial bearing characteristics are height $\hat{h}_{0}=2 \times 10^{-4} \mathrm{~m}$, radius $\hat{r}_{0}=0.02 \mathrm{~m}$, pressure $\hat{P}=1 \times 10^{5} \mathrm{~Pa}$, stator mass $1 \mathrm{Kg}$ with fluid properties $\hat{\rho}=800 \mathrm{Kgm}^{-3}$ and $\mu=$ $0.1 \mathrm{Kgm}^{-1} \mathrm{~s}^{-1}$. The resulting dimensionless stiffness and damping parameters are $K_{z}=55.56, D_{a}=1$ and dimensionless slip length $l_{s}=0.05$, based on a typical surface roughness.

The pressure distribution in a steady bearing, axial rotor amplitude $\epsilon=0$, is given in Figure 3 for a $\mathrm{PCB}$ and $\mathrm{NCB}$ in the case of a wide and narrow annulus. For the cases of a PCB and NCB the corresponding parallel bearing $(\beta=0)$ has internal and external pressurisation, respectively. In the case of a $\mathrm{NCB}$ and a PCB with a narrow annulus $(a=0.8)$ the pressure increase and decreases monotonically. However, for a $\mathrm{PCB}$ with a wide annulus the pressure decrease monotonically only in the case of a parallel faces $(\beta=0)$. Increasing the magnitude of the coning angle for both a PCB and a NCB gives a decrease in the pressure at a given radial position.

To compute the probability of contact $\left(P_{c}\right)$, an ensemble of deterministic realisations of the bearing behaviour are required giving the parameter values at 
$\left.a_{1}\right)$

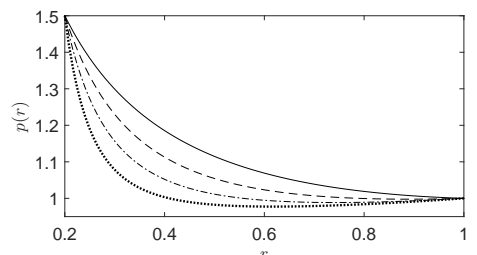

$\left.a_{2}\right)$

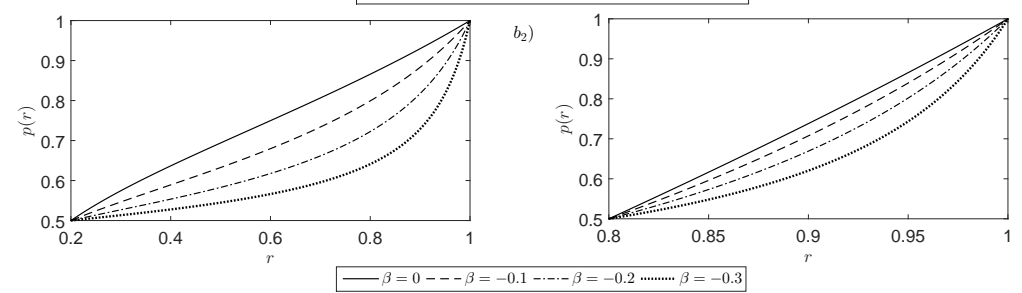

$\left.b_{1}\right)$

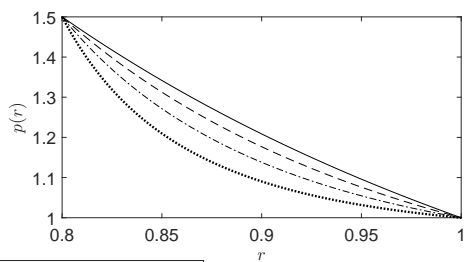

-.-- $\beta=0.2 \cdots \cdots \cdots \cdots \cdot \beta=0.3$ bearing width for a) wide $a=0.8$ and b) narrow $a=0.8$ annulus; $\lambda=0.1065, \sigma=36$, $\alpha=2.22$.
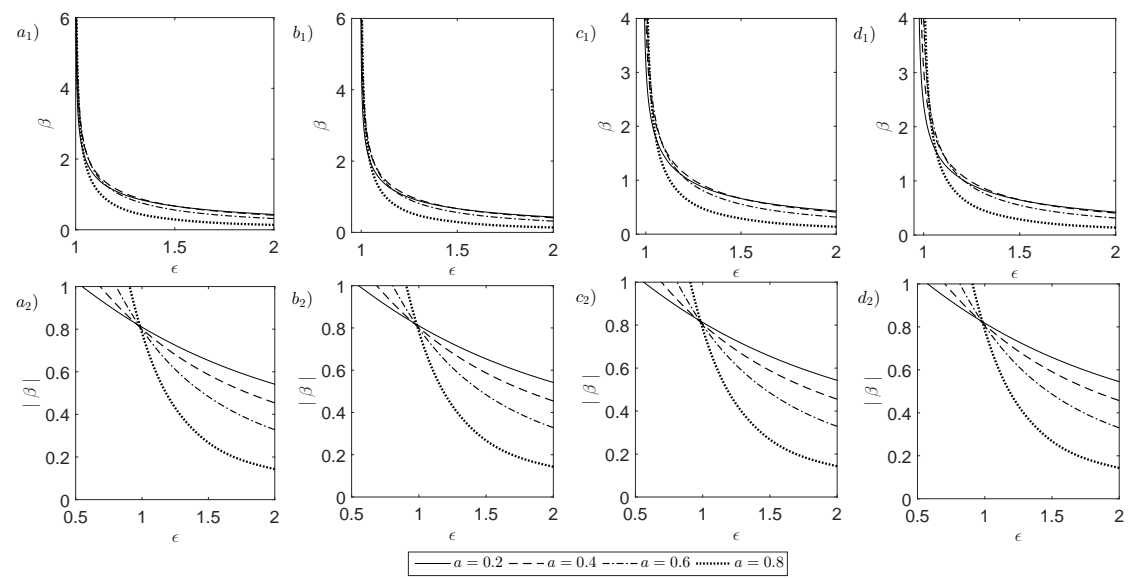

Figure 4: Coning angle $\beta$ and axial rotor amplitude $\epsilon$ associated with face contact in the case of a $\mathrm{PCB}\left(a_{1}\right.$ to $\left.d_{1}\right)$ and $\operatorname{NBC}\left(a_{2}\right.$ to $\left.d_{2}\right)$ with a) $\lambda=0$, b) $\lambda=0.1065$, c) $\lambda=0.45$, d) $\lambda=1$ and increasing inner radius $0.2 \leq a \leq 0.8 ; \sigma=36, \alpha=2.22$. 

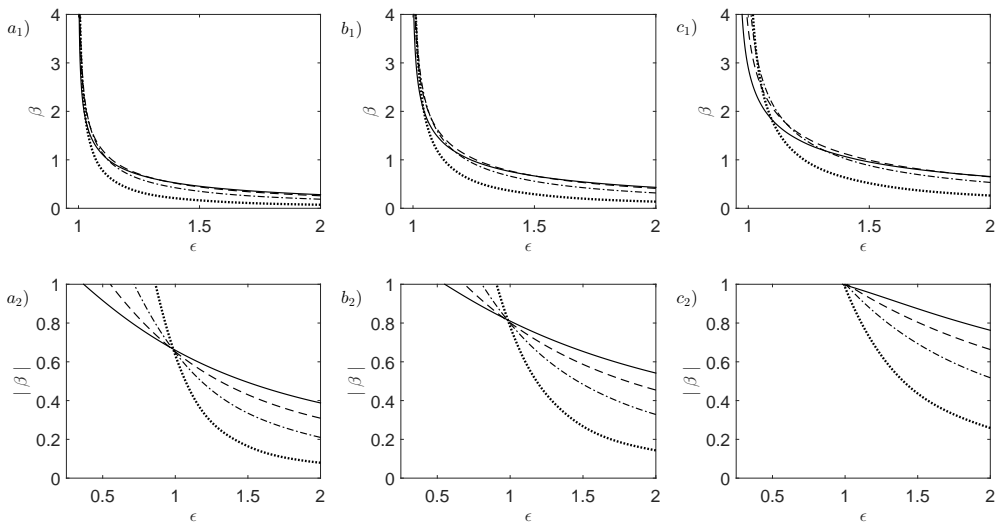

$a=0.2---a=0.4-\cdots-\cdot a=0.6 \cdots \cdots \cdots a=0.8$

Figure 5: Coning angle $\beta$ and axial rotor amplitude $\epsilon$ associated with face contact in the case of a PCB $\left(a_{1}-c_{1}\right)$ and $\operatorname{NBC}\left(a_{2}-c_{2}\right)$ with a) $\hat{h}_{0}=8 \times 10^{-5} \mathrm{~m}, \hat{r}_{0}=0.85 \mathrm{~m}, \lambda=0.6132$, $\sigma=33.18, \alpha=5.12$, b) $\hat{h}_{0}=2 \times 10^{-5} \mathrm{~m}, \hat{r}_{0}=0.02 \mathrm{~m}, \lambda=0.1065, \sigma=36, \alpha=2.22$ and c) $\hat{h}_{0}=5 \times 10^{-4} \mathrm{~m}, \hat{r}_{0}=0.048 \mathrm{~m}, \lambda=0.01923, \sigma=40.64, \alpha=1.0035$ and increasing inner radius $0.2 \leq a \leq 0.8$.

which face contact occurs. Values of the coning angle and axial rotor amplitude $\epsilon$ for which contact first occurs are given in Figure 4 for a PCB and NCB with increasing inner radius. Increases in the speed parameter are equivalent to rotational speeds of $0 \mathrm{rpm}, 2 \times 10^{4} \mathrm{rpm}, 4 \times 10^{4} \mathrm{rpm}$ and $6 \times 10^{4} \mathrm{rpm}$. In each Figure face contact will occur for parameter choices above these limiting curves; for parameter choices under the curve a fluid film is maintained within the bearing. The contact plots for a PCB and NCB display notably different characteristics; a NCB can have contact at significantly smaller values of $\epsilon$ than a PCB for adequately large coning angles. For a sufficiently small $\epsilon$ and coning angle a NCB has contact at smaller coning angles than for a PCB, however for a sufficiently large values the behaviour is reversed.

The values of the $\epsilon$ and $\beta$ at contact, depends markedly upon the bearing width and speed parameters. For a given value of coning angle in a PCB contact occurs at smaller $\epsilon$ as the bearing width $(1-a)$ decreases.

However, at sufficiently large values of $\beta$, a bearing of width $(1-a)=0.8$ has contact at smaller $\epsilon$ than $(1-a)=0.6$ (see values of $\beta \sim 1$ and $a=0.2$, 0.4 , in Figure 4, a1) to d1)). In a NCB, for coning angle $|\beta| \leq 0.81$ contact occurs at smaller $\epsilon$ as the bearing width decreases in contrast to coning angles $|\beta| \geq 0.82$ where contact occurs at larger $\epsilon$ as the bearing width decreases (see Figure 4, a2) to d2)). Increasing the speed parameter predicts contact at larger $\epsilon$ and $|\beta|$ with values dependent on the bearing width.

Companion plots showing values of $|\beta|$ and $\epsilon$ at first contact for the case of increasing inner radius, i.e. decreasing bearing width, and maintaining the same aspect ratio for consistency are displayed in Figure 5. Note the plots 
in Figure 5b) correspond to the those in Figure $4 \mathrm{~b}$ ). As can be observed in Figure 5 , reducing the characteristic height $\hat{h}_{0}$, and therefore increasing the characteristic radius $\hat{r}_{0}$ gives contact occurring at smaller $\epsilon$ and coning angles, whereas increasing the characteristic height and decreasing the characteristic radius gives contact at larger values.

Figures 4 and 5 identify for a bearing with conical deformation the possibility of face contact, dependent on both operational and design parameters.

\subsection{Uncertainty in coning angle}

To analyse close proximity of the rotor and stator the coning angle $\beta$ is taken as a random variable reflecting the typical lack of knowledge in its precise value. The resulting uncertainty on the magnitude of the minimum bearing gap $g_{\min }$ is of interest and in particular the probability of face contact. On taking the value of the coning angle as fixed but not known with certainty, a subjective interpretation of probability is obtained from expressing the coning angle as a random variable and implementing the method of derived distributions provides the corresponding distribution of variability in the bearing dynamics.

The coning angle is modelled by a double truncated normal function, see Bailey et al. [15], ensuring the angle is bounded by zero and magnitude of the angle is limited to $|\beta|=1$; thus guaranteeing the lubrication condition holds. Initially the average value of the coning angle is taken as $|\beta|=0.25$ and the standard deviation as $\sigma_{\epsilon}=0.05$, with the effect of changing the average value and standard deviation examined later. To ensure that the desired average value of the distribution remains representative due to its truncation, the prescribed mean of the distribution is chosen such that the effective median $M_{\beta}$ represents the required average value.

The probability density function (pdf) of $g_{\min }$ can be computed using the change of variables $f_{\text {Gmin }}\left(g_{\min }\right)=\left|\partial \beta / \partial g_{\min }\right| f_{\gamma}(\beta)$ when considering the coning angle as a random variable; $\partial \beta / \partial g_{\min }$ is calculated through the stroboscopic map solver. To compute the cumulative distribution function (CDF) of $g_{\min }$ the set of coning angles for which $g_{\min }$ is less than or equal to every possible value is required, $A_{\beta g_{\min }}=\left\{\gamma: \chi_{\epsilon}(\beta) \leq g_{\min }\right\}$. In this case $\chi_{\epsilon}$ represents the relation between the coning angle and $g_{\min }$ found using the deterministic mathematical model for different values of $\epsilon$. The $P_{c}$ is given by the CDF when $g_{\text {min }}=0$.

The untruncated CDF of $g_{\min }$ is given by

$$
\begin{aligned}
\tilde{F}_{G_{\text {min }}}\left(g_{\text {min }}\right) & =P\left(G_{\min } \leq g_{\min }\right)=P\left(\chi_{\epsilon}(\gamma) \leq g_{\min }\right)=P\left(\left\{\gamma: \chi_{\epsilon}(\beta) \leq g_{\min }\right\}\right) \\
& =\int_{A_{\beta g_{m i n}}} \tilde{f}_{\gamma}\left(\beta ; \mu_{\beta}, \sigma_{\beta}\right) d \beta
\end{aligned}
$$

with the truncated CDF found by normalising (6) within the probability space.

The result of $g_{\min }$ is confirmed by using the method of derived distribution to compute the CDF and compared to a direct Monte Carlo simulation; a comparison is shown in Figure 6. An increased number of simulations (stochastic realisations) are used in the Monte Carlo method and the CDF with $95 \%$ confidence bounds are computed where the required number of coning angle values 

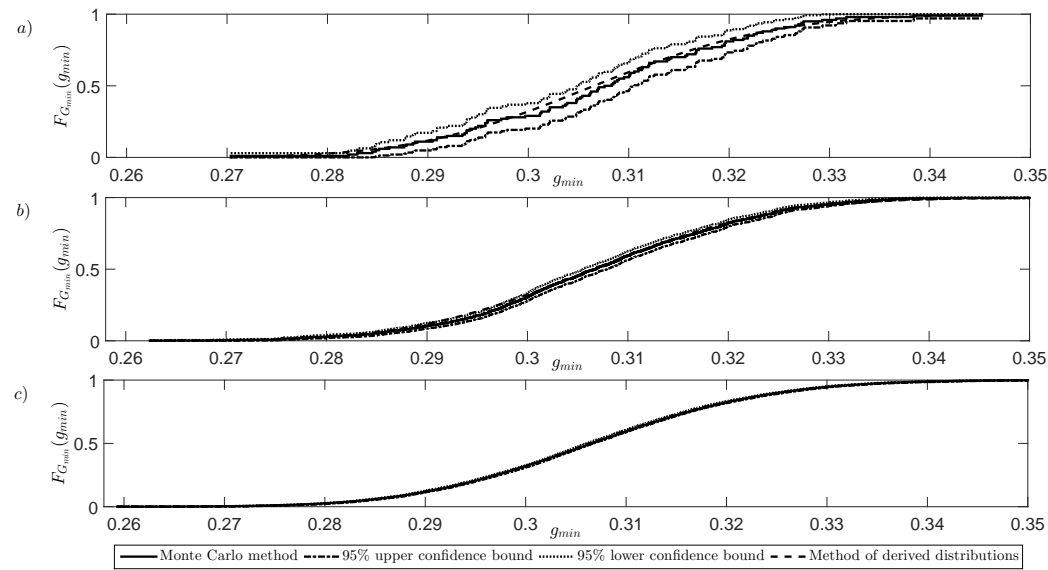

Figure 6: CDF of $g_{\min }$ computed with a) 100, b) 1000 and c) 10000 realisations of the MonteCarlo method as well the method of derived distributions in the case of a PCB with a wide annulus and coning angle distribution $M_{\beta}=0.25, \sigma_{\beta}=0.05 ; a=0.2, \lambda=0.1065, \sigma=36$, $\alpha=2.22$.

are taken from the truncated distribution at random. The stroboscopic map solver computes the corresponding values of $g_{\min }$ for each value of the coning angle used in the corresponding Monte Carlo calculation. The CDF is found via the method of derived distributions using the deterministic relationship between the coning angle and $g_{m i n}$, requiring the set $A_{\beta g_{m i n}}$, which is then used in equation (6). Increasing the number of Monte Carlo realisations gives the CDF converging to the solution obtained by the method of derived distributions; the output for the two methods is effectively the same for 10000 realisations in the Monte Carlo method. However, to achieve the required level of accuracy, the method of derived distributions is considerably less computationally expensive than the Monte Carlo method. The bearing configuration in Figure 6 gives $g_{\text {min }} \geq 0.259$, thus giving $P_{c}=0$. The results reported in the remaining of this manuscript are obtained by the method of derived distributions, due to its numerical efficiency.

Figure 7 shows the pdf of the coning angle, deterministic relationship between the coning angle and $g_{\min }$ along with the pdf and CDF of $g_{\min }$ in the case of increasing $\epsilon$ for a PCB and NCB with coning angle distribution; median $\left|M_{\beta}\right|=0.25$, standard deviation $\sigma_{\beta}=0.05$. The deterministic results show for increasing magnitude of coning angle, $g_{\min }$ decreases and as $\epsilon$ increases, $g_{\min }$ decreases for a given coning angle. According with the deterministic relations reported in Figure $\left.4 b_{1}\right)$ and $b_{2}$ ), it can be observed that for the operation conditions considered in Figure 7, a PCB has no contact for $\epsilon=1.0$ and a NBC has contact at $|\beta|=0.780$ whereas for $\epsilon=1.5$ a PCB has contact at $\beta=0.285$ and $|\beta|=0.265$ for a NCB. Using the deterministic relation between the coning angle and $g_{\min }$ allows the pdf of $g_{\min }$ to be computed, showing smaller values 
a)
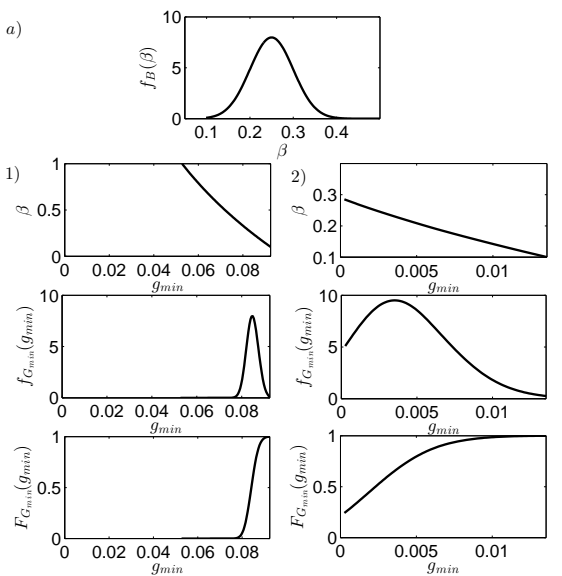
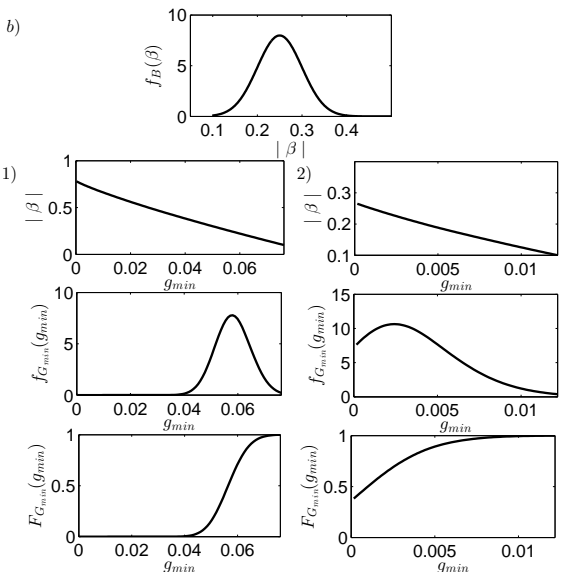

Figure 7: Pdf of coning angle $\beta$, deterministic relationship between $g_{\min }$ and coning angle $\beta$ and pdf and CDF of $g_{\min }$ in the case of increasing axial rotor amplitude $\epsilon 1$ ) $\epsilon=1.0$ and 2) $\epsilon=1.5$ in the case of a) PCB and b) NCB with narrow faces and coning angle distribution with median $\left|M_{\beta}\right|=0.25$, standard deviation $\sigma_{\beta}=0.05 ; a=0.8, \lambda=0.1065, \sigma=36$, $\alpha=2.22$.

of $g_{\min }$ are likely to occur as $\epsilon$ increase. The value of $P_{c}$ is given when the CDF intercepts the y-axis, giving $P$ (contact) $=0$ for $\epsilon=1.00$, given in columns 1 ) of Figure 7 , but in the case of $\epsilon=1.5$ it is found that the $P$ (contact) $=0.242$ for a PCB and $P$ (contact) $=0.382$ for a NCB, shown in columns 2) in Figure 7 .

It is advantageous to calculate $P_{c}$ directly for the parameter study using the relation

$$
P(\text { contact })=\frac{\int_{A_{\beta g_{t o l}}} \tilde{f}_{\gamma}\left(\beta ; \mu_{\beta}, \sigma_{\beta}\right) d \beta}{\tilde{F}_{\gamma}\left(b ; \mu_{\beta}, \sigma_{\beta}\right)}
$$

where $g_{t o l}$ denotes a given tolerance for $g_{\text {min }} ; g_{t o l}=0$ corresponds to contact between the bearing face. The set $A_{\beta g_{t o l}}=\left\{\gamma: \chi_{\epsilon}(\beta) \leq g_{t o l}\right\}$ comprises discrete values of the coning angle at which contact between the rotor and stator occurs for deterministic values of $\epsilon$, giving the $P_{c}$ as a function of $\epsilon$. The deterministic contact plots in Figure 4 and Figure 5 are used to compute the set $A_{\beta g_{t o l}}$.

Figure 8 gives $P_{c}$ against $\epsilon$ for increasing inner radius value $a$ for both a $\mathrm{PCB}$ and a NCB. For $\epsilon$ smaller than the equilibrium face clearance the $P_{c}$ is zero. Larger $\epsilon$ have an increased $P_{c}$ for increasing amplitude. A NCB has a larger $P_{c}$ than a $\mathrm{PCB}$ unless sufficiently large amplitudes of rotor oscillation are considered where a PCB has a larger $P_{c}$ than a NCB. Decreasing the inner radius value gives a decrease in the $P_{c}$, with a NCB in the case of a wide annulus $(a=0.2)$ having negligible $P_{c}$ for all amplitudes of rotor oscillations. Both a PCB and NCB have larger increases in the $P_{c}$ between narrower bearings ( $a=0.6$ to 0.8 ) than wider bearings $(a=0.2$ to 0.4$)$.

The effect of increasing the speed parameter on $P_{c}$ is illustrated in Figure 


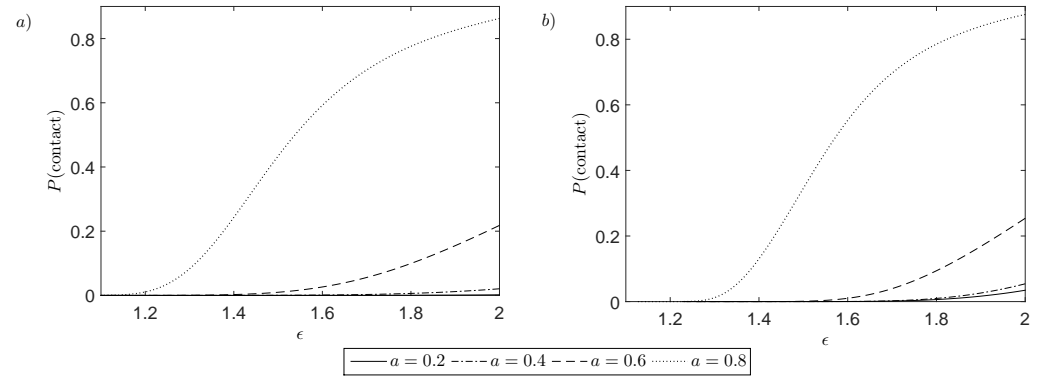

Figure 8: $P_{c}$ against axial rotor amplitude $\epsilon$ for increasing inner radius $0.2 \geq a \geq 0.8$ in the case of a) PCB and b) NCB with coning angle distribution $\left|M_{\beta}\right|=0.25, \sigma_{\beta}=0.1 ; \lambda=0.1065$, $\sigma=36, \alpha=2.22$.
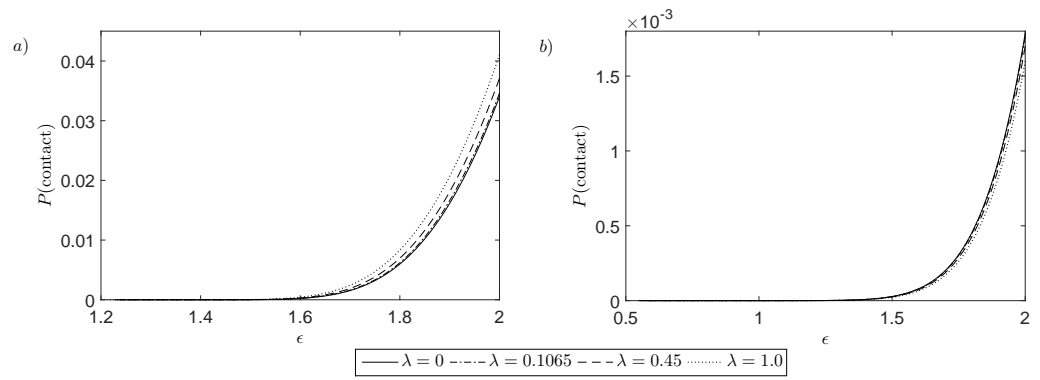

Figure 9: $P_{c}$ against axial rotor amplitude $\epsilon$ for increasing speed parameter $0 \leq \lambda \leq 1$ in the case of a) PCB and b) NCB with coning angle distribution $M_{\beta}=0.25, \sigma_{\beta}=0.1 ; a=0.2$, $\sigma=36, \alpha=2.22$. 


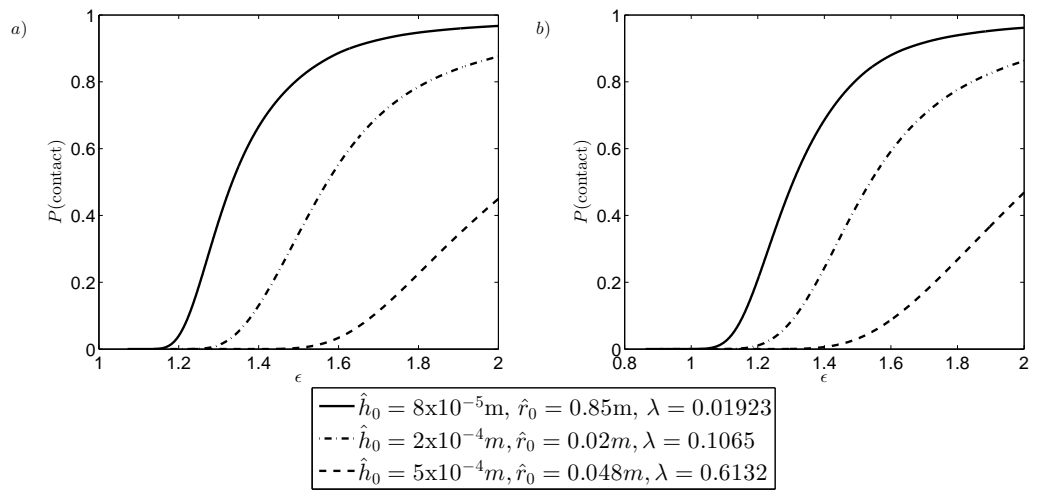

Figure 10: $P_{c}$ against axial rotor amplitude $\epsilon$ for various bearing geometries with the same aspect ratio and azimuthal speed in the case of a) $\mathrm{PCB}$ and b) NCB with coning angle distribution $\left|M_{\beta}\right|=0.25, \sigma_{\beta}=0.1 ; a=0.8, \sigma=36, \alpha=2.22$.

9 against $\epsilon$ in the case of a PCB and a NCB identifying a very small effect. Only the case of a wide bearing, $a=0.2$ is presented as the effect of the speed parameter in narrower bearings is almost negligible. Increasing $\epsilon$ increases $P_{c}$ whilst increasing the speed parameter has a small effect on a PCB and almost none for a NCB. From a practical point of view the effect of the speed parameter on $P_{c}$ can be completely neglected.

In Figure 10, for a $\mathrm{PCB}$ and $\mathrm{NCB}$, additional evaluation of $P_{c}$ is examined for different bearing geometries but maintaining the same aspect ratio and azimuthal speed. The value of $P_{c}$ increases with $\epsilon$ whilst increasing the characteristic height $\hat{h}_{0}$ and thus decreasing the characteristic radius $\hat{r}_{0}$ causes the $P_{c}$ to decrease. In this case, the $P_{c}$ for both cases, $\mathrm{PCB}$ and $\mathrm{NCB}$, have similar behaviour with a NCB having a slightly larger probability for a given amplitude of rotor oscillation.

The effect on $P_{c}$ from the probabilistic parameters of conical deformation are examined by considering 1) fixed standard deviation and increasing median and 2) fixed median with increasing standard deviation and displayed in Figure 11 for a PCB and a NCB. Increasing the median value increases the value of $P_{c}$, as shown in $\left.11 a_{1}\right)$ and $\left.11 a_{2}\right)$, such that coning angle with median $\left|M_{\beta}\right|=0.5$ has $P($ contact $)=1$ for $\epsilon \geq 1.6$ in both a PCB and NCB. A PCB has a smaller $P_{c}$ than a NCB in the case of $\left|M_{\beta}\right|=0.5$ and decreasing the median value gives a similar trend except at sufficiency large $\epsilon$ where a NCB has a smaller $P_{c}$ than a $\mathrm{PCB}$. Increasing the standard deviation of the coning angle increases the value of $P_{c}$ until a critical value $\epsilon_{c}$ where the $P_{c}$ curves intersect, see Figure 11b $b_{1}$ ) and $\left.b_{2}\right) ; \epsilon_{c}=1.57$ for a PCB and $\epsilon_{c}=1.54$ for a NCB. For the smallest standard deviation $\sigma_{\beta}=0.01$ the $P_{c}$ increases from zero to one over a very small range of $\epsilon$ for both a PCB and NCB, almost as a smooth step function. Generally by increasing the standard deviation $\sigma_{\beta}$ the corresponding CDFs become flatter with higher values of $P_{c}$ for $\epsilon<\epsilon_{c r}$ and smaller for $\epsilon>\epsilon_{c r}$. 

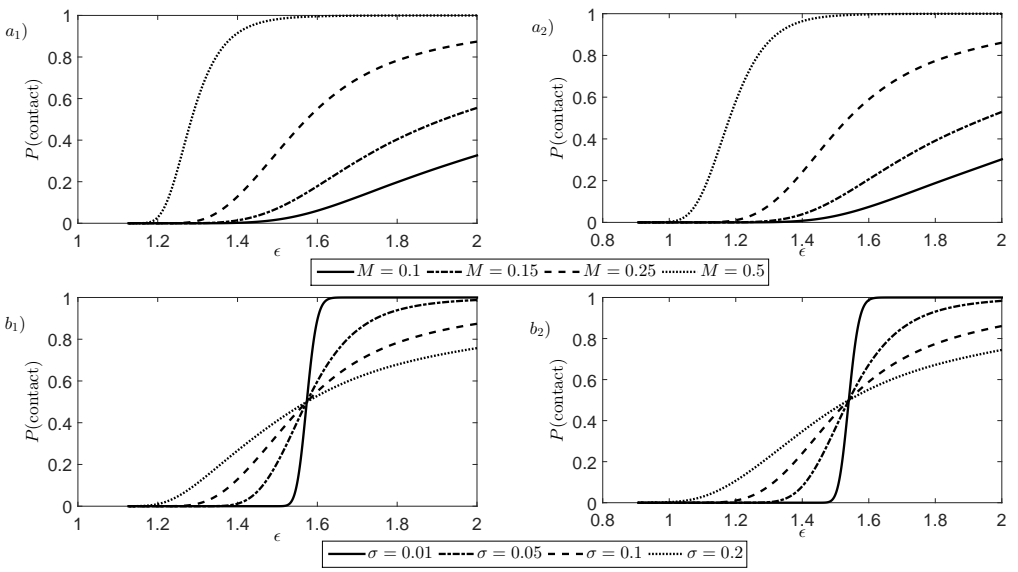

Figure 11: $P_{c}$ against axial rotor amplitude $\epsilon$ for 1) fixed standard deviation $\sigma_{\beta}=0.1$ and increasing median $0.1 \leq\left|M_{\beta}\right| \leq 0.5$ and 2) fixed median $\left|M_{\beta}\right|=0.25$ and increasing standard deviation $0.01 \leq \sigma_{\beta} \leq 0.2$ in the case of a) PCB and b) NCB; $\lambda=0.1065, a=0.8, \sigma=36$, $\alpha=2.22$.

\subsection{Uncertainty in coning angle and amplitude of the rotor oscillation $\epsilon$}

Uncertainty in the dynamics of the bearing can also arise due to the bearing experiencing excitations. Hou et al. [12] presented a simplified model for the excitations where the oscillations are taken to be periodic with a random amplitude.

This approach is adopted through incorporating axial rotor oscillations which are assumed to be periodic and have a given amplitude with a value that is not exactly known and considered a random variable. Taking the coning angle and amplitude of the rotor oscillations as uncertain, results in a two parameter random input problem. Use of deterministic relation between $|\beta|$ and $\epsilon$ enables an exact evaluation of $P_{c}$ to be computed, via the method of derived distribution. In this case the rotor oscillations remain defined by $h_{r}=\epsilon \sin t$, with $\epsilon$ considered as a random variable. Distributions of $|\beta|$ and $\epsilon$ are described by a truncated normal distribution, ensuring $\epsilon$ is non-negative and has a restricted magnitude set at $\epsilon=2$, i.e. twice the equilibrium face clearance. To consider bearing operation under extreme conditions, initially the average of $\epsilon$ is taken to be $\epsilon=1.5$ with standard deviation $\sigma_{\epsilon}=0.2$.

The joint pdf of the coning angle and $\epsilon$ is given by the product of their marginal pdfs as $f_{\gamma, E}(\beta, \epsilon)=f_{\gamma}(\beta) f_{E}(\epsilon)$, i.e. statistically independent. Using the deterministic curves from Figures 4 and 5 to identify the set of coning angles $A_{\epsilon g_{t o l}}=\left\{\gamma: G_{\min } \leq g_{t o l}\right\}$ and amplitudes of rotor oscillations $A_{\epsilon g_{t o l}}=\{E$ : $\left.G_{\text {min }} \leq g_{\text {tol }}\right\}$ allows the $P_{c}$ to be calculated using

$$
\begin{aligned}
P\left(G_{\text {min }} \leq 0\right) & =P\left(\left\{\gamma: G_{\min } \leq g_{t o l}\right\}\right) P\left(\left\{E: G_{\min } \leq g_{t o l}\right\}\right) \\
& =\int_{A_{\beta g_{t o l}}} f_{\gamma}(\beta) d \beta \int_{A_{\epsilon g_{t o l}}} f_{E}(\epsilon) d \epsilon
\end{aligned}
$$




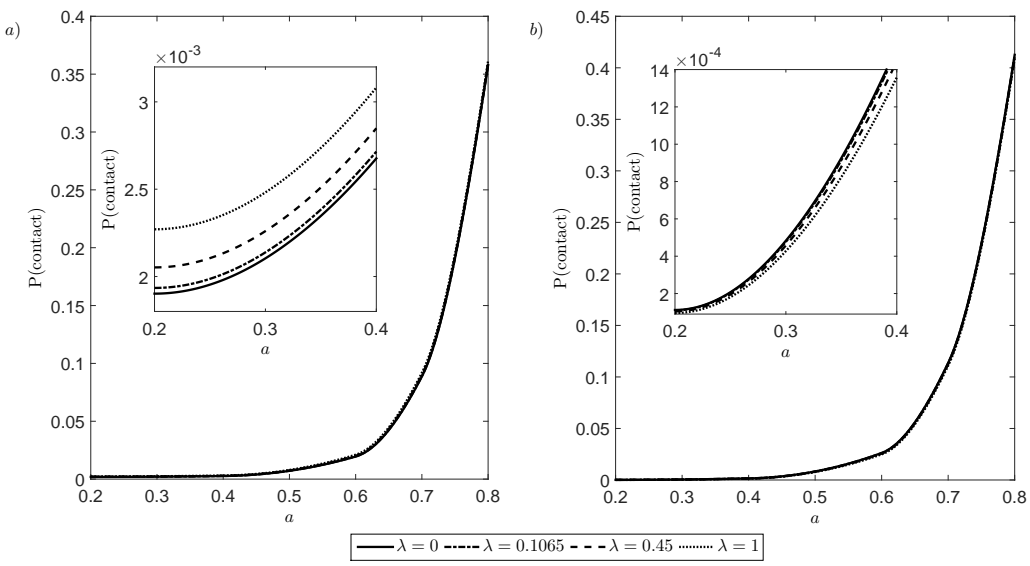

Figure 12: $P_{c}$ against decreasing bearing width $0.2 \leq(1-a) \leq 0.8$ for increasing speed parameter $0 \leq \lambda \leq 1$ in the case of a) $\mathrm{PCB}$ and b) $\mathrm{NCB}$ with coning angle distribution $\left|M_{\beta}\right|=0.25, \sigma_{\beta}=0.1$ and $\epsilon$ distribution $M_{\epsilon}=1.5, \sigma_{\epsilon}=0.2 ; \sigma=36, \alpha=2.22$.

Figure 12 shows the $P_{c}$ against decreasing bearing geometries in the case of a PCB and NCB for increasing speed parameter; as previously the speed parameter has negligible effect on the $P_{c}$. As previously mentioned, the bearing response to imposed rotor oscillations is defined by a complex interaction between the different parameters defining the problem; bearing geometry, rotor inclination angle, fluid pressurisation, slip condition and effects of inertia. In this work we are analysing two cases which arise from rotor deformation due to over pressurisation of the bearing; internal pressurisation relates to a $\mathrm{PCB}$, $\beta>0$, and external pressurisation to a $\mathrm{NCB}, \beta<0$. In the case of internal pressurisation, the effects of centrifugal inertia and pressure gradient both act on the flow in the same radial direction and therefore increasing the effects of inertia results in an increase of the fluid radial velocity and concurrently a reduction in the loading capacity. On the other hand, in the case of external pressurisation, the effects of centrifugal inertia and pressure gradient act in opposite directions, resulting in a decrease of fluid radial velocity and increase of the loading capacity in the case of increasing effects of inertia (for more detail see the results reported in Table 1 of reference [8]). More significantly, increasing the slip length results in the radial velocity increasing and the load capacity decreasing for both cases. This effect becomes stronger as the minimum gap decreases, reaching a limit condition where the slip effect becomes dominant with respect to all other effects, increasing the possibility of face contact. This is the physical explanation of the unexpected result in Figures 9 and 12, i.e. the speed parameter has negligible effect on the probability of contact.

The effect of different bearing geometries on the $P_{c}$ for increasing bearing widths is shown in Figure 13 for a PCB and NCB (note the curves for $\hat{h}_{0}=$ $2 \times 10^{-4} \mathrm{~m}$ are the same as the plots in Figure 12 for $\left.\lambda=0.1065\right)$. Decreasing the 
a)
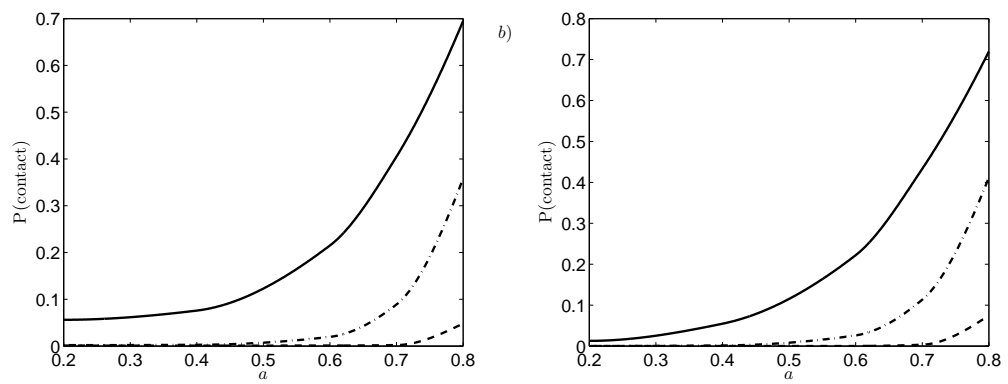

$-\hat{h}_{0}=8 \times 10^{-5} \mathrm{~m}, \hat{r}_{0}=0.85 \mathrm{~m}, \lambda=0.01923$

$\cdots \hat{h}_{0}=2 \times 10^{-4} m, \hat{r}_{0}=0.02 m, \lambda=0.1065$

$---\hat{h}_{0}=5 \times 10^{-4} m, \hat{r}_{0}=0.048 m, \lambda=0.6132$

Figure 13: $P_{c}$ against decreasing bearing width $0.2 \leq(1-a) \leq 0.8$ for various bearing geometries in the case of a) PCB and b) NCB with coning angle distribution $\left|M_{\beta}\right|=0.25$, $\sigma_{\beta}=0.1$ and $\epsilon$ distribution $M_{\epsilon}=1.5, \sigma_{\epsilon}=0.2 ; \sigma=36, \alpha=2.22$.

bearing width gives an increase in the $P_{c}$, whereas increasing the characteristic height $\hat{h}_{0}$ and decreasing the characteristic radius $\hat{r}_{0}$ to maintain the same aspect ratio, decreases the $P_{c}$. In the case of the largest characteristic height $\hat{h}_{0}=$ $5 \times 10^{-4} \mathrm{~m}$ a $\mathrm{PCB}$ has a smaller $P_{c}$ than a NCB, whereas smaller characteristic heights $\hat{h}_{0}=2 \times 10^{-4}, \hat{h}_{0}=8 \times 10^{-5}$ give a PCB having a larger $P_{c}$ than a NCB for $a \leq 0.469, a \leq 0.558$, respectively, otherwise the situation is reversed.

The effect of the probabilistic parameters of the conical deformation on the $P_{c}$, for fixed standard deviations and increasing medians as well as fixed medians and increasing standard deviations are shown in Figure 14 for a PCB and NCB. Increasing the median values of both $|\beta|$ and $\epsilon$ gives an increase in the $P_{c}$, see Figure $\left.14 a_{1}\right)$ and $b_{1}$ ). The larger median values of the coning angle give a non-zero $P_{c}$ for amplitudes of rotor oscillations less than the equilibrium face clearance. For $\left|M_{\beta}\right|=0.1$ a PCB and NCB have effectively the same $P_{c}$ over all medians of $\epsilon$, but for larger values of $\left|M_{\beta}\right|$ a NCB has a larger $P_{c}$ than a $\mathrm{PCB}$, with the difference increasing with the median value.

For a sufficiently small standard deviation $\sigma_{\epsilon} \leq 0.145$, the $P_{c}$ increases as the standard deviation of the coning angle increases. Whereas for sufficiently large standard deviation $\sigma_{\epsilon} \geq 0.374$, the $P_{c}$ decreases as the standard deviation of the coning angle increases. For $0.145 \leq \sigma_{\epsilon} \leq 0.374$ there is crossover between the different values. A NCB has a larger $P_{c}$ than a PCB for a given standard deviation of the coning angle and $\epsilon$.

To consider possible design control in order to reduce the probability of contact, we analyse the effects of changing the values of the stiffness and damping coefficients of the system. Figure 15a) reports values of $P_{c}$ against increasing the median of $\epsilon$ in the case of a NCB, for three different values of the dimensionless stiffness parameter; one corresponding to the value used in the previous analysis, $K_{z}=55.56$, and the other two $10 \%$ and $20 \%$ larger than the original 
$a_{1}$

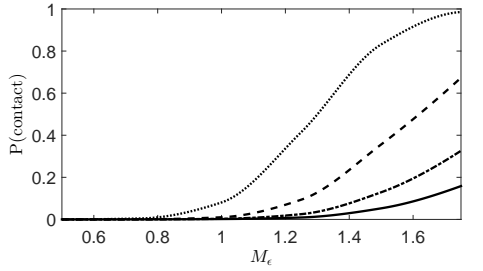

$\left.b_{1}\right)$

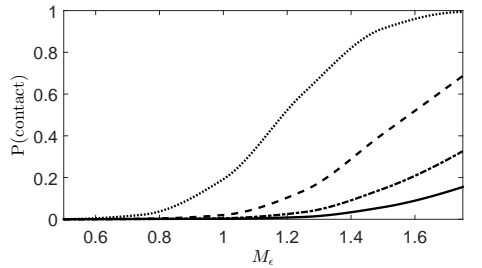

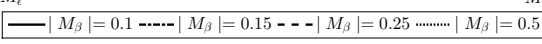

$\left.a_{2}\right)$

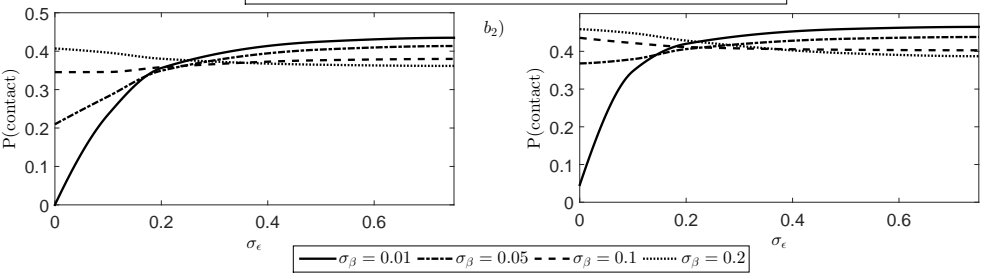

Figure 14: $P_{c}$ against decreasing bearing width $0.2 \leq(1-a) \leq 0.8$ for various bearing geometries in the case of a) PCB and b) NCB for 1) fixed standard deviation $\sigma_{\beta}=0.1, \sigma_{\epsilon}=0.2$ and increasing median $0.1 \leq\left|M_{\beta}\right| \leq 0.5,0.5 \leq M_{\epsilon} \leq 1.75$ and 2) fixed median $\left|M_{\beta}\right|=0.25$, $M_{\epsilon}=1.5$ and increasing standard deviation $0.01 \leq \sigma_{\beta} \leq 0.2,0 \leq \sigma_{\epsilon} \leq 0.75 ; a=0.8$.

a)

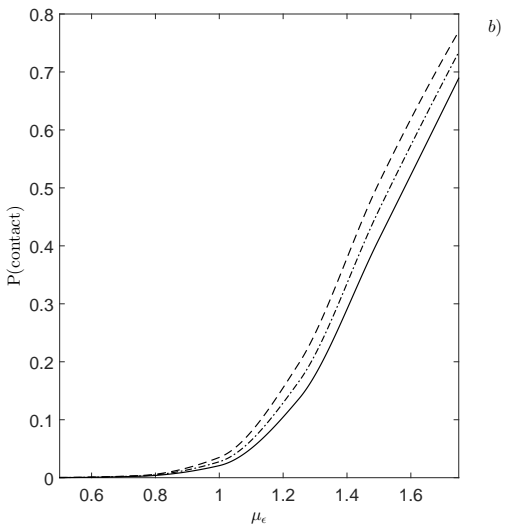

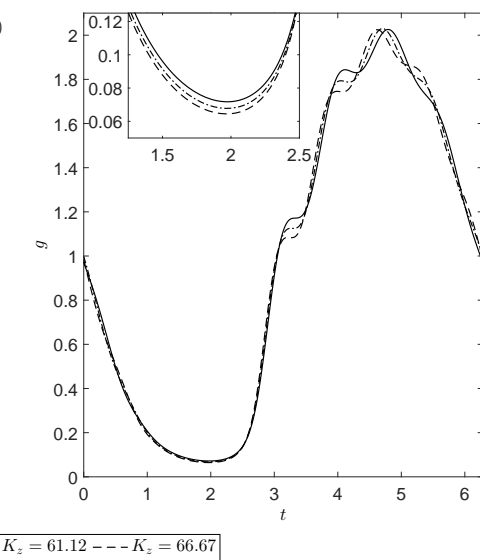

Figure 15: In the case of a $\mathrm{NCB}$ a) $P_{c}$ against increasing median of $\epsilon 0.5 \leq \epsilon \leq 1.75$ for increasing stiffness parameter $55.56 \leq K_{z} \leq 66.67$ with coning angle distribution $\left|M_{\beta}\right|=0.25$, $\sigma_{\beta}=0.1$ and $\sigma_{\epsilon}=0.2$ and b) deterministic minimum face clearance over the time period NCB for $\epsilon=1, \beta=-0.2 ; \sigma=36, \alpha=2.22$. 
value. Results show that decreasing the value of $K_{z}$ reduces the probability of contact for all median values of $\epsilon$. This situation arises because the stator opposes less resistance to the fluid force induced by the axial displacement of the rotor by decreasing the spring stiffness parameter $K_{z}$, resulting in a larger minimum face clearance for a given $\epsilon$, as shown in the deterministic dynamic plot in Figure 15b), consequently reducing the probability of contact. Similar plots are achieved when examining a PCB. Corresponding plots for increasing values of the structural damping $D_{a}$ show the structural damping has an insignificant effect on the probability of contact. Therefore in terms of engineering design, the $P_{c}$ can be reduced or controlled by decreasing the magnitude of the stiffness of the stator $K_{z}$. However, this approach can be only considered under structures limitations, since smaller values of $K_{z}$ also implies reduction on the rigidity of the system.

Our results show contact always occurs at the side of higher pressure, where the minimum face clearance arises according to the corresponding conical angle, i.e. at the inner radius of the bearing when $\beta>0$ and the outer radius when $\beta<0$. This result is consistent with the experimental observations in Jing et al. [20], where spiral groove noncontacting seals are studied, indicating that the pressure deformation decreases the seal clearance at the side of a mechanical face seal with higher pressure, which means face wear occurs more seriously at the side with high pressure than the side with low pressure.

It is important to mention that the present analysis is indicative of what can happen during the dynamic motion of a bearing that has been destabilised by some periodic external condition. As commented above, over pressurisation of the bearing can induce angular bearing deformation, which in our analysis is considered to be small enough to ensure the lubrication approximation remains valid, where $\hat{\beta}=\delta_{0} \beta$ with $\beta$ as our scaled angle. Near contact, due to the geometric distortion, this condition no longer holds and the validity of the Reynolds equation can be questionable. However, this is probably the only alternative to analyse the near contact condition using classical engineering approaches, unless molecular dynamics is considered, since it could be argued that in the limit of near contact even the continuous formulation of the problem is violated.

\section{Conclusions}

A simplified mathematical model is introduced for a deterministic highly rotating coned bearing with the rotor and stator having slip velocity boundary conditions. An incompressible fluid flow model is derived to model the pressurised fluid flow and is coupled to the axial motion of the stator, which is modelled as a spring-mass-damper system. The coupled system can be represented by a single second order non-autonomous ordinary differential equation in terms of the time dependent minimum face clearance from which periodic solutions are found numerically using a stroboscopic map solver.

The effect of uncertain values for the coning angle parameter, arising due to possible rotor deformations by the pressurized flow, as well as the parameter modelling the axial rotor amplitude are examined; these uncertain parameters 
are taken as random variables. Considering the coning angle as a random variable allows extended analysis for the pdf of the minimum face clearance $g_{\min }$, $\mathrm{CDF}$ of $g_{\min }$ and the probability of contact $\left(P_{c}\right)$. Analysis shows the $P_{c}$ increases due to an increase in amplitude of axial rotor oscillations $(\epsilon)$, reduction in the bearing width as well as in bearing set-ups which have a fixed aspect ratio, for a decreased characteristic height/increase characteristic radius. The speed parameter has a negligible effect on the $P_{c}$, however the speed parameter can have a significant effect on the bearing dynamics. Increasing the median of the coning angle random variable gives an increase in the $P_{c}$ where as increasing the standard deviation gives a non-monotonic trend; for amplitude of rotor oscillation below a critical value $\epsilon_{c}$ the $P_{c}$ increases whereas for amplitude of rotor oscillations $\epsilon>\epsilon_{c}$ the $P_{c}$ decreases.

Accounting for possible external excitations on the bearing results in the coning angle and axial rotor amplitude $\epsilon$ being considered as random variables. The $P_{c}$ is calculated using the method of derived distributions with results indicating decreasing the bearing width as well as configurations with smaller characteristic height, and larger radius to maintain the same aspect ratio, have an increase in the $P_{c}$. The speed parameter gives a minimal change in the magnitude of the $P_{c}$. Increasing the median of the coning angle and $\epsilon$ increases the $P_{c}$, whereas increasing the standard deviation of the random variables gives a non-monotonic trend. The $P_{c}$ is affected insignificant by the structural damping, however the $P_{c}$ may be reduced or controlled through decreasing the magnitude of the stator stiffness.

\section{Funding}

This work was supported by funding from the EPSRC Doctoral Prize grant No. EP/M506588/1.

\section{Appendix A. Derivation Equations}

Expressions for $A$ and $B$ are defined as

$$
\begin{aligned}
& A\left(g, \lambda, l_{s}, \beta\right)=\left(1-a^{2}\right)\left(p_{I}-p_{a}\right)+2\left(p_{O}-p_{I}\right) \frac{G_{I}}{G(g, 1)} \\
&+2 \lambda\left(L_{I}-\frac{L(g, 1)}{G(g, 1)} G_{I}\right), \\
& B\left(g, l_{s}, \beta\right)=\sigma\left(H_{I}-\frac{H(g, 1)}{G(g, 1)} G_{I}\right) .
\end{aligned}
$$


Expressions for $G, H$ and $L$ are given by

$$
\begin{aligned}
G(g, r)= & \frac{1}{g-a \beta+6 l_{s}}\left(\frac{1}{(g-a \beta)^{2}} \ln \left(\frac{g r}{a(g+(r-a) \beta}\right)+\frac{1}{g-a \beta}\left(\frac{1}{g}-\frac{1}{g+(r-a) \beta}\right)\right. \\
& \left.+\frac{1}{36 l_{s}^{2}}\left(\ln \left(\frac{g\left(g+(1-a) \beta+6 l_{s}\right)}{(g+(1-a) \beta)\left(g+6 l_{s}\right)}\right)+6 l_{s}\left(\frac{1}{g+(r-a) \beta}-\frac{1}{g}\right)\right)\right), \\
H(g, r)= & \frac{1}{\beta^{2}}\left(\left(\frac{1}{g}-\frac{1}{g+(r-a) \beta}\right)\right. \\
& \left.+\frac{g-a \beta+6 l_{s}}{36 l_{s}^{2}}\left(\ln \left(\frac{g\left(g+(1-a) \beta+6 l_{s}\right)}{(g+(1-a) \beta)\left(g+6 l_{s}\right)}\right)+6 l_{s}\left(\frac{1}{g+(r-a) \beta}-\frac{1}{g}\right)\right)\right), \\
L(g, r)= & \frac{r^{2}-a^{2}}{2}+\frac{l_{s}}{\beta^{2}}\left(\frac{4}{3} l_{s}\left(g-a \beta+2 l_{s}\right)\left(\frac{1}{g+(r-a) \beta+2 l_{s}}-\frac{1}{g+2 l_{s}}\right)\right. \\
& +\frac{3}{2}\left(g-a \beta+6 l_{s}\right) \ln \left(\frac{\left(g+6 l_{s}\right)\left(g+(r-a) \beta+2 l_{s}\right)}{\left(g+2 l_{s}\right)\left(g+(r-a) \beta+6 l_{s}\right)}\right) \\
& \left.-\frac{14}{3} l_{s} \ln \left(\frac{g+(r-a) \beta+2 l_{s}}{g+2 l_{s}}\right)\right),
\end{aligned}
$$

respectively. Expressions for a NCB are also derived of similar form and for equations in the case of a no-slip bearing, see [4].

Expressions $G_{I}, H_{I}$ and $L_{I}$ are given by

$$
\begin{aligned}
G_{I}= & \frac{1}{g-a \beta+6 l_{s}}\left(-\frac{1}{2(g-a \beta)^{2}} \ln \left(\frac{a(g+(1-a) \beta)}{g}\right)+\frac{(1-a)(g-a \beta-\beta)}{2 \beta g((g-a \beta)}\right. \\
& +\frac{1}{72 \beta^{2} l_{s}^{2}}\left(6 l_{s}(1-a) \beta\left(\frac{g-a \beta-\beta}{g}\right)\right. \\
& \left.\left.\quad+\left(\left(g-a \beta+6 l_{s}\right)^{2}-\beta^{2}\right) \ln \left(\frac{g\left(g+(1-a) \beta+6 l_{s}\right)}{(g+(1-a) \beta)\left(g+6 l_{s}\right)}\right)\right)\right), \\
H_{I}= & \frac{1}{\beta^{2}}\left(-\frac{(1-a)(2 g-(1+a) \beta)}{2 \beta g}+\frac{3\left(g-a \beta+2 l_{s}\right)}{2 \beta^{2}} \ln \left(\frac{g+(1-a) \beta}{g}\right)\right. \\
& +\frac{g-a \beta+6 l_{s}}{72 \beta^{2} l_{s}^{2}}\left(\frac{6 l_{s}(1-a) \beta(g-(1+a) \beta)}{g}\right. \\
& \left.\left.\quad+\left(\left(g-a \beta+6 l_{s}\right)^{2}-\beta^{2}\right) \ln \left(\frac{g\left(g+(1-a) \beta+6 l_{s}\right)}{(g+(1-a) \beta)\left(g+6 l_{s}\right)}\right)\right)\right),
\end{aligned}
$$




$$
\begin{aligned}
& L_{I}= \frac{\left(1-a^{2}\right)^{2}}{8}+\frac{2 l_{s}{ }^{2} a\left(1-a^{2}\right)}{3 \beta\left(g+2 l_{s}\right)}+\frac{l_{s}^{2}}{2 \beta^{2}}\left(8 a+1-9 a^{2}\right)-\frac{4(1-a) l_{s}^{2}}{\beta^{3}}\left(g+5 l_{s}\right) \\
&+\ln \left(\frac{g+6 l_{s}}{g+(1-a) \beta+6 l_{s}}\right)\left(-\frac{3 l_{s} a\left(1-a^{2}\right)}{4 \beta} \frac{3 l_{s}}{4 \beta^{2}}\left(g+6 l_{s}\right)\left(1-3 a^{2}\right)+\frac{9 l_{s} a}{4 \beta^{3}}\left(g+6 l_{s}\right)^{2}\right. \\
&\left.-\frac{3 l_{s}}{4 \beta^{4}}\left(g^{3}+18 l_{s} g^{2}+108 l_{s}^{2} g+216 l_{s}^{3}\right)\right) \\
&+\ln \left(\frac{g+(1-a) \beta+2 l_{s}}{g+2 l_{s}}\right)\left(-\frac{3 l_{s} a\left(1-a^{2}\right)}{4 \beta} \frac{l_{s}}{12 \beta^{2}}\left(9 g+26 l_{s}\right)\left(1-3 a^{2}\right)\right. \\
&-\frac{l_{s} a}{4 \beta^{3}}\left(9 g^{2}+\right.\left.\left.52 g l_{s}+68 l_{s}^{2}\right)+\frac{l_{s}}{4 \beta^{4}}\left(3 g^{3}+26 l_{s} g^{2}+68 l_{s}^{2} g+56 l_{s}^{3}\right)\right) .
\end{aligned}
$$

Expressions for a NCB are also derived of similar form and for equations in the case of a no-slip bearing, see [4].

\section{References}

[1] Etison I. Dynamic analysis of noncontacting face seals. Transactions of the ASME 1982;104:460-468.

[2] Green I, Etison I, Stability threshold and steady-state response of noncontacing coned-face seals. ASLE Trans. 1985; 28:449-460.

[3] Green I. The rotor dynamic coefficient of coned-face mechanical seals with inward or outward flow. Journal of Lubrication Technology;1983: 105, 297302.

[4] Bailey NY, Cliffe KA, Hibberd S, Power H. On the dynamics of a high speed coned fluid lubricated bearing. IMA Journal of Applied Mathematics 2014;79:535-561.

[5] Miksis MJ, Davis SH. Slip over rough and coated surfaces. Journal of Fluid Mechanics 1994;273:125-139.

[6] Sarkar K, Prosperetti A. Effective boundary conditions for Stokes flow over a rough surface. Journal of Fluid Mechanics1996;316:223-240.

[7] Bailey NY, Cliffe KA, Hibberd S, Power H. Dynamics of a parallel, highspeed, lubricated thrust bearing with Navier slip boundary conditions. IMA Journal of Applied Mathematics 2015;80:1409-1430.

[8] Bailey NY, Cliffe KA, Hibberd S, Power H. Dynamics of a high speed coned thrust bearing with a Navier slip boundary condition. Journal of Engineering Mathematics 2015; doi:10.1007/s10665-015-9793-y.

[9] Kalita W, Rodkiewicz CzM, Kennedy JS. On the laminar flow characteristic of conical bearing. Part IAnalytical approach. Journal of Tribology $1986 ; 108: 53-58$ 
[10] Kalita W, Rodkiewicz CzM, Kennedy JS. On the laminar flow characteristic of conical bearing. Part IIExperimental Verification Journal of Tribology 1986;108:59-64.

[11] Lee AS, Green I. An experimental investigation of the steady state response of noncontacting flexible mounted rotor mechanical face seal. Journal of Tribology 1995;117:153-159.

[12] Hou, Z, Zhou Y, Dimentberg MF, Noori M. A stationary model for periodic excitation with uncorrelated random disturbances. Probabilistic Engineering Mechanics 1996;11:191-203.

[13] Zigang L, Jun J, Zhui T. Non-linear vibration of an angular-misaligned rotor system with uncertain parameters. Journal of Vibration and Control 2014;doi:10.1177/1077546314525432.

[14] Benaroya H, Han SM, Nagurka M. Probabilistic models for dynamical systems. CRC Press: Taylor \& Francis Group; 2013.

[15] Bailey NY, Cliffe KA, Hibberd S, Power H. Probability of Face Contact for a High-Speed Pressurised Liquid Film Bearing Including a Slip Boundary Condition. Lubricants 2015;3:493-521.

[16] Garratt JE, Cliffe KA, Hibberd S, Power H. Centrifugal inertia effects in high speed hydrostatic air thrust bearings. Journal of Engineering Mathematics 2012;76:59-80.

[17] Zhang G, Zhao W. Design and Experimental Study on the Controllable High-Speed Spiral Groove Face Seals. Tribology Letters 2014;53:497-509.

[18] Pan Y, Bhushan B, Maali A. Slip length measurement of confined air flow on three smooth surfaces. Langmuir 2013;29:4298-4302.

[19] Zou M, Davan J, Green I. Feasibility of Contact Elimination of a Mechanical Face Seal Through Clearance Adjustment. Transactions of the ASME 2000;122:478-484.

[20] Jing X, Xudong P, Shaoxian B, Xiangkai M, Jiyun L. Experiment on Wear Behavior of High Pressure Gas Seal Faces. Chinese Jounal of Mechnical Engineering 2014;27:1287-1293. 